\title{
BOCK, Hans Manfred, MEYER KALKUS, Reinhart, TREBISCH, Michel, Entre Locarno et Vichy. Les relations culturelles franco-allemandes dans les années 1930
}

Christophe Duhamelle et Patrice Veit

\author{
(2) OpenEdition \\ Journals \\ Édition électronique \\ URL : http://journals.openedition.org/ifha/2051 \\ DOI : 10.4000/ifha.2051 \\ ISSN : 2198-8943 \\ Éditeur \\ IFRA - Institut franco-allemand (sciences historiques et sociales) \\ Référence électronique \\ Christophe Duhamelle et Patrice Veit, «BOCK, Hans Manfred, MEYER KALKUS, Reinhart, TREBISCH, \\ Michel, Entre Locarno et Vichy. Les relations culturelles franco-allemandes dans les années 1930 ", Revue \\ de l'IFHA [En ligne], Date de recension, mis en ligne le 01 janvier 1994, consulté le 22 septembre 2020. \\ URL : http://journals.openedition.org/ifha/2051 ; DOI : https://doi.org/10.4000/ifha.2051
}

Ce document a été généré automatiquement le 22 septembre 2020.

(C)IFHA 


\title{
BOCK, Hans Manfred, MEYER KALKUS, Reinhart, TREBISCH, Michel, Entre Locarno et Vichy. Les relations culturelles franco-allemandes dans les années 1930
}

\author{
Christophe Duhamelle et Patrice Veit
}

Cet ouvrage foisonnant est issu d'un colloque organisé en décembre 1990 par l'office allemand d'échanges universitaires (DAAD) et l'Institut d'histoire du temps présent (CNRS). S'attachant à la période comprise entre la crise de 1929 et la dernière décennie avant la Seconde Guerre Mondiale, se situant donc de part et d'autre de 1933, le livre aborde une phase cruciale des relations culturelles entre la France et l'Allemagne dont il tente de cerner et mettre en lumière la complexité. Il prend en compte les niveaux les plus divers, officiels, officieux, individuels, et s'interroge sur le statut et le rythme propres des relations culturelles dans l'ensemble des relations internationales. Ayant pour visée une histoire sociale des relations culturelles, cet ouvrage offre une multiplicité de regards qui vont des politiques culturelles aux institutions bilatérales, des échanges les plus divers, qu'ils soient universitaires, scientifiques, religieux et philosophiques aux réseaux de sociabilité, des représentations de l'autre dans la littérature et l'édition, les arts et l'anthropologie à la circulation des mythes et des stéréotypes nationaux.

46 contributions constituent cet ensemble, qui sont autant d'études de cas, regroupées en trois grandes parties. La première concerne la culture et le champ politique: elle aborde les équivoques du rapprochement franco-allemand, dont l'évolution de la coopération franco-allemande qui va du cercle de Sohlberg (1930) au Comité FranceAllemagne (1934) offre un exemple; l'entente franco-allemande au regard du pacifisme et de l'idée européenne dans leurs diverses variations; l'importance des milieux et des réseaux chrétiens de part et d'autre du Rhin. La seconde partie s'intéresse aux relations 
et aux échanges dans les milieux universitaires et scientifiques: les organismes d'échange, en analysant principalement le rôle du bureau parisien du DAAD créé en 1930; les romanistes allemands et les germanistes français; les relations dans le domaine des sciences, entre historiens français et allemands; l'expérience de l'Allemagne chez Raymond Aron et Jean-Paul Sartre; enfin, l'anthropologie et les théories raciales dans les relations franco-allemandes. La troisième partie s'attache aux divers lieux et milieux en prenant d'abord en considération des médiations culturelles entre la France et l'Allemagne à l'exemple de Carl Einstein dans le domaine des arts et de la littérature, des échanges dans le milieu du cinéma, des éditions Grasset entre 1930 et 1939 ou de la revue Europe, en étudiant ensuite le champ littéraire - Thomas Mann et ses représentations de la France, Georges Bernanos et sa réception de l'Allemagne et du nazisme ou bien encore la réception de Céline dans l'Allemagne nazie -, pour finir par les émigrés allemands en France.

D'une grande diversité dans les approches et les interrogations, ces actes que complète une importante bibliographie en fin de volume, sont une contribution fondamentale à la compréhension de la période de l'entre-deux-guerres.

Christophe DUHAMELLE, Patrice VEIT 